\title{
Effect of particle morphology on mechanical properties of liquid marbles
}

Saeid Azizian, ${ }^{1 *}$ Syuji Fujii,,${ }^{2,3 *}$ Moe Kasahara, ${ }^{4}$ Hans-Jürgen Butt,${ }^{5}$ and Michael Kappl ${ }^{5}$

${ }^{1}$ Department of Physical Chemistry, Faculty of Chemistry

Bu-Ali Sina University, Hamedan 65167, Iran

${ }^{2}$ Department of Applied Chemistry, Faculty of Engineering

Osaka Institute of Technology, 5-16-1 Omiya, Asahi-ku, Osaka, 535-8585, Japan

${ }^{3}$ Nanomaterials Microdevices Research Center

Osaka Institute of Technology, 5-16-1, Omiya, Asahi-ku, Osaka 535-8585, Japan

${ }^{4}$ Division of Applied Chemistry, Graduate School of Engineering

Osaka Institute of Technology, 5-16-1, Omiya, Asahi-ku, Osaka 535-8585, Japan

${ }^{5}$ Max Planck Institute for Polymer Research, Ackermannweg 10, D-55128 Mainz, Germany

\section{*Corresponding Authors}

E-mail: sazizian@basu.ac.ir; Phone +98-81-3828-2807; Fax +98-81-1838-0709

E-mail: syuji.fujii@oit.ac.jp; Phone +81-6-6954-4274; Fax +81-6-6957-2135 


\section{ABSTRACT}

In order to better understand the influence of the shape of solid particles on the stability of liquid marbles, we investigated liquid marbles stabilized by hydrophobized calcium carbonate particles with spherical and rod-shaped morphologies. Static properties, such as the effective surface tension, and the dynamic behavior i.e. the compression-decompression features for several cycles of the liquid marbles were investigated. Liquid marbles stabilized with spherical $\mathrm{CaCO}_{3}$ particles show an elastic response to mechanical deformation almost up to collapse. In contrast, liquid marbles prepared with rod-like particles exhibit a more plastic response to compression. It is concluded that the main differences in behavior of the prepared liquid marbles arise from how the solid particles can arrange/orient at the air/water interface.

KEYWORDS: Liquid Marble, $\mathrm{CaCO}_{3}$, Elasticity, Plasticity, Hydrophobic particles.

\section{INTRODUCTION}

Liquid marbles are typically millimeter-sized liquid droplets stabilized by solid particles adsorbed at the gas-liquid interface. The particles encapsulate the liquid held in place by capillary forces. Liquid marbles have striking properties that cannot be attained using bare liquid droplets [1-7]. Because of their non-wetting property due to the encapsulating particulate stabilizer, liquid marbles exhibit low friction, which allows them to move easily on solid substrates and on liquid surfaces. Contamination of the encapsulated liquid can be avoided because the inner liquid is isolated from any substrates. Liquid marbles can be divided, merged, disrupted and levitated, and their contents (the encapsulated liquid phase) can be mixed and released on demand. Therefore, 
liquid marbles have gained increasing interest in view of their potential applications in cosmetics [8,9], transport \& microfluidics [10-12], miniature reactors [13-15], personal \& health care products [16], sensors [17], accelerometers [18], pressure-sensitive adhesives [19], and actuators [20].

Particle properties including size and the hydrophilic-hydrophobic balance of the surface are important to determine formability and stability of the liquid marbles. Size of liquid marble stabilizer should be important to determine liquid marble size and shape. It has been clarified that hydrophobic solid particles, which repel water, can adsorb to droplet surfaces and work as an effective liquid marble stabilizer [21]. In contrast, on hydrophilic particle beds water is taken up by capillary action and no liquid marble is formed. Furthermore, it is possible to control the liquid marble stability using stimuli-responsive particles whose hydrophobic-hydrophobic balance can be tuned by external stimuli $[6,22,23]$. The particle shape should be also important to determine formability and stability of the liquid marble. However, little is known about the influence of particle shape on liquid marble stability [24].

The elasticity and robustness of liquid marbles are crucial properties to be investigated, because in applications they are exposed to external mechanical stress, and were investigated theoretically and experimentally, recently [25-28]. In this study, the effect of morphology of liquid marble stabilizer, namely sphere and rod, on mechanical properties of liquid marbles is investigated using compression / decompression cycles between parallel plates. The mechanical properties are correlated with the structure of the encasulatingshell formed by the different solid particles.

\section{EXPERIMENTS}


Both spherical and rod-shaped $\mathrm{CaCO}_{3}$ nanoparticles (Figures 1 and $\mathrm{S} 1$ ) were kindly donated from Shiraishi Kogyo Kaisha, Ltd (Hyogo, Japan). These nanoparticles were used as a liquid marble stabilizer, which were synthesized by carbonation process in aqueous medium $[29,30]$. Numberaverage diameter of the spherical $\mathrm{CaCO}_{3}$ particles $\left(\mathrm{C}_{1}\right)$ was determined to be $80 \mathrm{~nm}$ from transmission electron microscopy studies $(n=200)$. The density was measured to be $2.52 \mathrm{~g} / \mathrm{cm}^{3}$ by a helium pycnometer. Number-average sizes of long and short axes of rod-shaped particles $\left(\mathrm{C}_{2}\right)$ were determined to be $2.8 \pm 1.4 \mu \mathrm{m}$ and $0.8 \pm 0.4 \mu \mathrm{m}$, respectively $(n=200)$. (The rod-shaped particle sample did also contain sphere-like particles with a diameter of less than $1 \mu \mathrm{m}$. Unfortunately, a further purification of this sample to obtain purely rod-shaped particles was not possible. Octadecanoic acid was adsorbed to the nanoparticle surfaces via acid-base interaction between the carboxylic acid and $\mathrm{CaCO}_{3}$ to render the $\mathrm{CaCO}_{3}$ nanoparticles hydrophobic. Both the spherical and rod-shaped $\mathrm{CaCO}_{3}$ nanoparticles have strong tendency to agglomerate and therefore existed as flocs. Gellan gum (Kelcogel F) was kindly donated from CPKelco.

The liquid marbles ( $\mathrm{L}_{1}$ and $\mathrm{L}_{2}$ ) were prepared by rolling $15 \mu 1$ droplets of deionized water on the hydrophobic $\mathrm{CaCO}_{3}$ powders $\left(\mathrm{C}_{1}\right.$ and $\left.\mathrm{C}_{2}\right)$ for approximately $10 \mathrm{~s}$. To test the mechanical properties of the liquid marbles, a home-made setup was used. The liquid marbles were compressed between two parallel glass slides while measuring both the applied force and the induced deformation (Scheme 1). The setup consisted of a solid support for the lower glass slide, a motorized Z-stage for vertical movement (HVM130-30-HDS, Owis, Staufen, Germany) that was carrying a force sensor (GSO-10, Transducer Techniques, Temecula, CA, USA) to which the upper glass slide was attached. The force sensor output signal was amplified (TMO-2 signal conditioner, Transducer Techniques, Temecula, CA, USA) and recorded by a computer using an AD-card (NI PCI-6251, National Instruments Corporation, Austin TX, USA). The same computer was also used to control 
the movement of the Z-stage via PCI motor controller card (Owis PS30 PCI card, Owis, Staufen, Germany). Both the movement of the Z-stage and the recording of the force signal were controlled by a self-written Labview program (National Instruments Corporation, Austin, TX, USA). Calibration of the force sensor was done by adding known weights to the upper glass slide holder and recording the corresponding voltages from the signal conditioner. As the deflection of the force sensors at loads of $1 \mathrm{mN}$ is only in the order of $1 \mu \mathrm{m}$, it can be safely be neglected compared to the deformation of the liquid marbles which for such forces was in the order of millimeters. The standard speed of compression in our experiments was $11 \mu \mathrm{m} / \mathrm{s}$, using a step size of $8.1 \mu \mathrm{m}(1000$ encoder steps) between each force reading. During compression tests, the liquid marbles were imaged from top through the upper glass slide using a video microscope that consisted of a Navitar 600 zoom lens system (Navitar Inc. Rochester, NY, USA), a Mitutoyo 2x M-Plan Apo Objective (Mitutoyo Corporation, Kawazaki, Japan) and an USB camera (Ueye UI22805E-M-GL, IDS GmbH, Obersulm, Germany).

Scanning electron microscopy (SEM; VE-8800, 12 kV, Keyence, Osaka, Japan) studies were conducted on dried samples previously sputter-coated (SC-701 Quick Coater, Elionix, Tokyo, Japan) with $\mathrm{Au}$. This SEM was used for observation of $\mathrm{CaCO}_{3}$ particles. Liquid marbles prepared using Gellan gum were observed by SEM before and after compression using a tackiness tester (TAC1000, Rhesca Co.). The probe (SUS, a diameter of $5 \mathrm{~mm}$ ) was brought onto contact with the liquid marbles placed on SUS substrate at $10 \mathrm{~mm} / \mathrm{s}$. After a constant contact time $(30 \mathrm{~s})$, the probe was brought up at $10 \mathrm{~mm} / \mathrm{s}$. The push distance and applied pressure were $0.25 \mathrm{~mm}$ and $<2497 \mathrm{~Pa}$, respectively. 


\section{RESULTS and DISCUSSIONS}

\subsection{Characterization and compression of liquid marbles}

Individual liquid marbles were fabricated by rolling a $15 \mu \mathrm{L}$ water droplet over the dried $\mathrm{CaCO}_{3}$

powders (Figure 1). Both the spherical and rod-shaped powders immediately coat the water drops and render them both hydrophobic and non-wetting. These liquid marbles could be transferred onto a glass slide. Significant roughness was observed on their surfaces, which suggests that $\mathrm{CaCO}_{3}$ multilayers/flocs coated the water droplets, rather than just a monolayer. The weight ratios of water $/ \mathrm{CaCO}_{3}$ were gravimetrically calculated to be $96 / 4$ and $97 / 3$ for spherical and rod-shaped particle systems, respectively.

The effective surface tensions of the prepared liquid marbles $\left(\mathrm{L}_{1}\right.$ and $\left.\mathrm{L}_{2}\right)$ were measured by making marbles as large as possible [31]. Then they tend to flatten out and form more a puddle rather than a spherical object. The height of such a puddle is given by the surface tension and the contact angle. Measuring the puddle height $h_{\max }$ and by assuming the apparent contact angle to be close to $180^{\circ}$, the effective surface tension can be calculated by:

$$
\gamma=\frac{\rho g h_{\max }^{2}}{4}
$$

The height of both samples, as a function of their volumes, were measured and plotted in Figure S2. By using the data in Figure S2 and eq. (1), the effective surface tension values were estimated to be $60.0( \pm 2.5)$ and $41.5( \pm 2.0) \mathrm{mN} / \mathrm{m}$ for the spherical $\left(\mathrm{L}_{1}\right)$ and the rod-shaped $\left(\mathrm{L}_{2}\right) \mathrm{CaCO}_{3}$ liquid marbles, respectively. 
Figure $2 \mathrm{a}$ shows the typical result for a compression test at a compression speed of $11 \mu \mathrm{m} / \mathrm{s}$, plotted as compression force versus relative compression, for both liquid marbles stabilized by spherical $\left(\mathrm{L}_{1}\right)$ and rod-shaped particles $\left(\mathrm{L}_{2}\right)$. For comparison, the result for a pure water droplet of the same volume that was compressed between two super-hydrophobic glass slides [32] is also shown. During compression, the force increases monotonically up to a maximum force of $\approx 1.1$ $\mathrm{mN}$ where the liquid marbles collapse. At this point, the relative compression $\left(1-h / h_{0}\right)$ had reached more than $50 \%$, where $h_{0}$ is the initial height of the marble (at $t=0$ ) and $h$ the height given at any later time of the experiment. Up to relative compression of about $35 \%$, corresponding to a force of $\approx 0.3 \mathrm{mN}$, curves recorded with liquid marbles $\mathrm{L}_{1}$ and pure water drops are almost identical. For forces above $0.3 \mathrm{mN}$, the liquid marbles show a slightly higher stiffness than the water droplet. For forces of more than $0.7 \mathrm{mN}$, the water droplet started to move laterally between two superhydrophobic glass slides. The close similarity between these two curves indicates that the main resistance to compression for deformations of less than $35 \%$ stems from the increase in surface area and the surface tension of water.

Liquid marbles $\mathrm{L}_{2}$ show a different compression behavior. At low compressions, they start out with a higher slope, which levels out so that a shoulder-like shape was observed for compressions below $20 \%$. At deformations above $20 \%$, they show a similar force- $v s$-compression as $\mathrm{L}_{1}$. $\mathrm{L}_{2}$ reaches slightly higher maximal compression before collapse and they are more stable. Similar to $\mathrm{L}_{1}$, the forces to compress the LM are higher than force required to compress a pure water drop.

To further analyze the compression behavior, we imaged the liquid marbles through the compressing glass slides by video microscopy (Figures 2b,c). When increasing the compression force, the maximal diameter of the liquid marbles increases. At $\left(1-h / h_{0}\right)$ above 0.3 , macroscopic 
cracks appear. For $\mathrm{L}_{2}$ liquid marbles this point coincides with the endpoint of the observed shoulder in Fig.2a. By further increasing the compression force, the number and the size of these cracks increases until the liquid marble ruptures completely, typically at $\left(1-h / h_{0}\right)=0.6$.

\subsection{Elastic and plastic behavior of liquid marbles}

In order to investigate the elastic and the plastic behavior of the liquid marbles, compressiondecompression forces were measured for several consecutive cycles for the $L_{1}$ sample. In each cycle, the maximum load was less than half of the rupture force. At this load, cracks were already visible, but they disappeared again when the force was released. Cracks reappeared at the same locations when force was applied again. In Figure 3, the consecutive compression-decompression cycles are plotted as force versus the relative compression $\left(1-h / h_{0}\right)$ of the marble. The compressiondecompression plots overlay. Thus the spherical $\mathrm{CaCO}_{3}$ liquid marble $\left(\mathrm{L}_{1}\right)$ show mainly an elastic response to the applied deformation, even though the formation of cracks itself is certainly not elastic. Only a small irreversible compression is detected when plotting $h_{f} / h_{0}$ versus the cycle number (Fig. 3 inset). where, $h_{f}$ is the height of the marble after each compression. The height of the $\mathrm{L}_{1}$ liquid marble decreases by $5 \%$ in the first cycle, and then, decreases about $1.5 \%$ in each of the next cycles. The larger height reduction during the first compression cycle may in part due to flattening out of surface roughness of the particle multilayer and/or packing of flocs during first contact between upper glass slide and liquid marble.

When compressing $\mathrm{L}_{2}$ liquid marbles prepared with the rod-shaped $\mathrm{CaCO}_{3}$, repeatedly (Figure 4) a "shoulder" was observed in each compression, but not when decompressing the LM. The height of the marble decreased monotonically with each cycle. The compression-decompression curves at each cycle do not overlay, and also, the obtained plots for different cycles do not match 
to each other. Therefore, liquid marbles $\left(\mathrm{L}_{2}\right)$, prepared with rod-shaped $\mathrm{CaCO}_{3}$ are less elastic and show a larger portion of plastic deformation. The plastic deformation should be caused by compression of the rod-shaped $\mathrm{CaCO}_{3}$ particle layer with three dimensional structure on the liquid marbles (see section 3.3). A comparison between Figure 3 inset and Figure 4 inset shows that the height decrease for the rod-shaped $\mathrm{CaCO}_{3}$ liquid marble is twice of that for the spherical $\mathrm{CaCO}_{3}$ liquid marble. Thus, spherical $\mathrm{CaCO}_{3}$ leads to more elastic liquid marbles than the one prepared with rod-shaped particles. Plastic deformation of liquid marbles $\left(\mathrm{L}_{2}\right)$, prepared with rod-shaped $\mathrm{CaCO}_{3}$ depends on the compression/decompression rate. The higher the rate of compressiondecompression, the more elastic the process is (Figures 4 and S3). The plastic effect was most pronounced at the lowest rate. By decreasing the rates of the compression-decompression, the height of the liquid marble $\left(\mathrm{L}_{2}\right)\left(h / h_{0}\right)$ decreases to higher extent. This might be due to more time available for the particles to rearrange/reorient at the interface, when the applied force is ramped up slowly.

The rupture force of the liquid marble, where the liquid marble collapses and the inner liquid is ejected, is equal to $F=A_{c} \Delta p$, where $\Delta p$ is the Laplace pressure and $A_{c}$ is the contact area between the glass plate and the liquid marble. The Laplace pressure for a liquid marble compressed between two parallel plates is given by $\Delta p=2 \gamma|\cos \theta| / h, \gamma$ is the effective surface tension of the liquid marble, $\theta$ is its contact angle with glass plate and $h$ is the height of the liquid marble, The contact area $A_{c}$ at rupture is proportional to $V / h$, where $V$ is the liquid marble volume [33]. Therefore, the rupture force of the liquid marble increases with its volume, and it can be represented by using a power law empirical equation as well $\left(F=C_{0} V^{\alpha}\right.$, where $C_{0}$ and $\alpha$ are constants) [33]. The rupture forces for the liquid marbles $\left(\mathrm{L}_{1}\right.$ and $\left.\mathrm{L}_{2}\right)$ were measured as a function of their volumes. A plot (Figure 5) shows that indeed, for both samples $\left(\mathrm{L}_{1}\right.$ and $\left.\mathrm{L}_{2}\right)$, the rupture force increases with the 
liquid marble volume, as expected from the power law model [33]. The obtained $\alpha$ and $C_{0}$ values for $L_{1}$ and $L_{2}$ are $0.33,0.39$ and $0.34,0.36$, respectively. It should be noted that, as the volume of the liquid marble increases, its shape starts to flatten out due to gravity and becomes puddle like. Also, it was not possible to prepare stable $\mathrm{CaCO}_{3}$ liquid marbles with volumes higher than $400 \mu 1$, as they always collapsed already during handling.

\subsection{Structure of solid particles at the surface of liquid marbles}

In order to investigate the orientation of the $\mathrm{CaCO}_{3}$ particles at the air/water interface, SEM studies were conducted on the surface of the liquid marbles prepared from $2.0 \mathrm{wt} \%$ aqueous Gellan gum solution. A free-flowing aqueous solution of Gellan gum $\left(15 \mu \mathrm{L}, 80{ }^{\circ} \mathrm{C}\right)$ was placed on the $\mathrm{CaCO}_{3}$ powder bed and liquid marbles were formed by rolling the droplet at $25^{\circ} \mathrm{C}$. During the formation of the liquid marbles, the aqueous Gellan solution became to be gel, and the surfaces of these gelled liquid marbles were observed before and after compression followed by drying. Nearspherical shapes of the liquid marbles prepared using Gellan gum aqueous solution remained intact even after drying and morphological changes before and after the compression could be discussed.

Figures $6 \mathrm{a}$ and $6 \mathrm{~b}$ show the SEM images of the spherical $\mathrm{CaCO}_{3}$ samples $\left(\mathrm{C}_{1}\right)$ on the liquid marble before and after compression. These figures are similar to the SEM images of the original powder sample (FigureS1). Therefore, there is no specific morphological change in the spherical $\mathrm{CaCO}_{3}$ nanoparticles $\left(\mathrm{C}_{1}\right)$ at the water interface. Figures $6 \mathrm{c}$ and $6 \mathrm{~d}$ show the SEM images of the rod-shaped $\mathrm{CaCO}_{3}$ sample $\left(\mathrm{C}_{2}\right)$ on the liquid marble before and after the compression. Before the compression, the rod-shaped $\mathrm{CaCO}_{3}$ nanoparticles existed on the liquid marble surface with random orientations. Once the liquid marble was compressed, the rod-shaped $\mathrm{CaCO}_{3}$ nanoparticles reclined on the surface. As shown in Figure 2a, a shoulder appears in the force diagram, when the 
rod-shaped $\mathrm{CaCO}_{3}$ liquid marble $\left(\mathrm{L}_{2}\right)$ is compressed. Also, shown in Figure 4a, at consecutive cycles, the length of the shoulder decreases, while the required force for the observation of the shoulder increases. The possible interpretation for this observation is that the additional force was required to change the orientation of rod-shaped $\mathrm{CaCO}_{3}$ layer on the liquid marble surface upon the compression and the required force increased with repeated cycles because the packing degree of the rod-shaped nanoparticles increased.

\section{CONCLUSION}

Aqueous liquid marbles prepared with two different hydrophobic $\mathrm{CaCO}_{3}$ powders showed a different mechanical response to compression. The finding indicates that the structure of solid particles affects the dynamic behavior of liquid marbles. Liquid marbles coated with spherical $\mathrm{CaCO}_{3}$ nanoparticles showed nearly elastic compression/decompression force curves. These curves were almost fully reversible up to compression to $40 \%$ of the initial marble height. At such strain, the coating of the liquid marbles starts forming cracks. At this point the stress exceeds the Laplace pressure calculated with an effective surface tension close to that of water. Liquid marbles coated with rod-shaped $\mathrm{CaCO}_{3}$ microparticles showed a higher stiffness at low compression and a yielding at the onset of crack formation, indicated by a shoulder in compression-decompression cycles. Consecutive compression-decompression cycles lead to plastic deformation of the particle layer and a decrease of the resistance against mechanical deformation. The plastic response even at low compression in rod-shaped particle system and the elastic response in the spherical particle system indicate that the spherical particles rearrange easier and more reversible than the rodshaped nanoparticles at liquid marble surface. The rod-shaped particles form three dimensional 
network structure, and therefore, the liquid marbles stabilized with the particles show a higher resistance to deformation at low stress and more plastic deformation at higher stress. This is the first report on the effect of structure of solid particles on the dynamic behavior of liquid marbles, which has not been reported previously using the same technique (mechanical compression) [2528]. The present work opens a new sight for investigation of effect of other structures of solid particles and also the effect of particles mixture on the behavior of liquid marbles. 


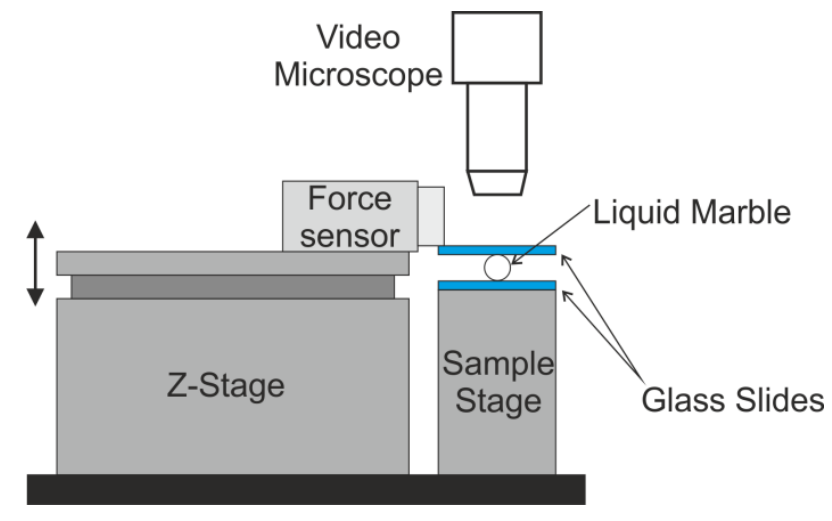

Scheme 1. Home-made apparatus designed to evaluate the mechanical property of liquid marbles.

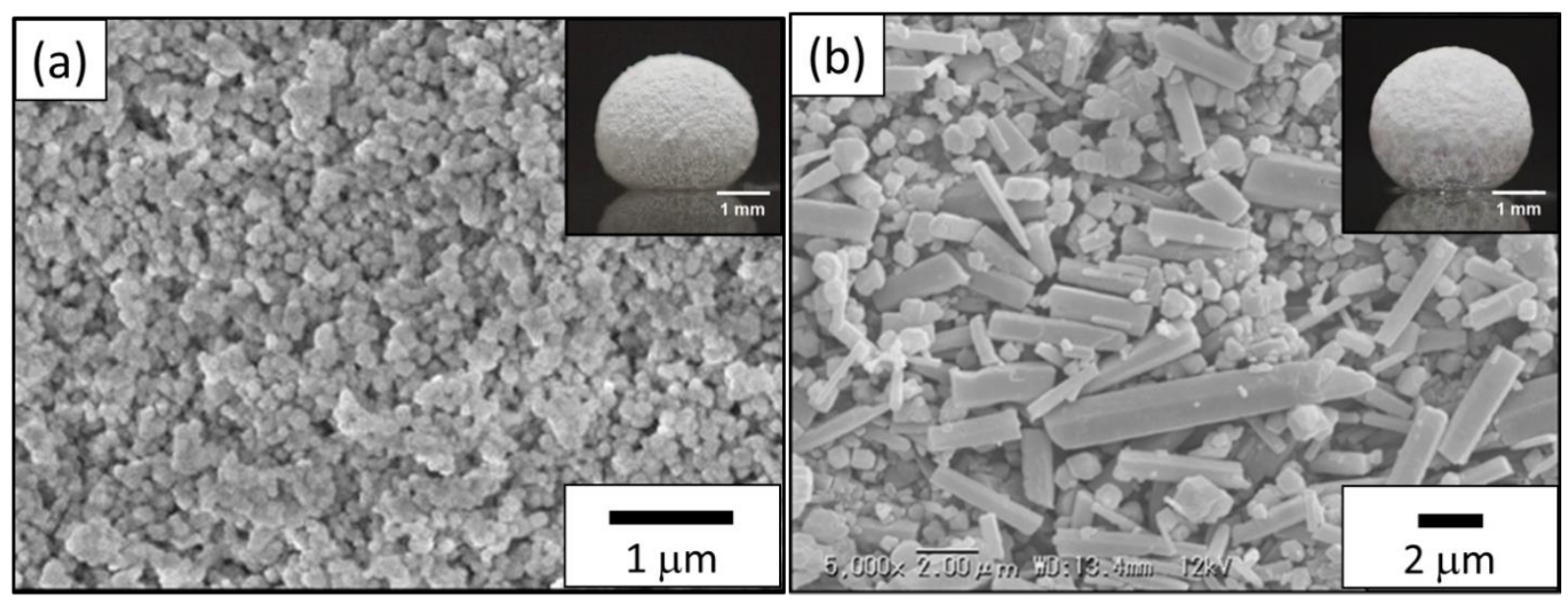

Figure 1. The SEM images of the prepared $\mathrm{CaCO}_{3}$ samples with different morphologies, (a) spherical nanoparticles and (b) rod-shaped particles. Insets are liquid marbles stabilized with the $\mathrm{CaCO}_{3}$ particles. 

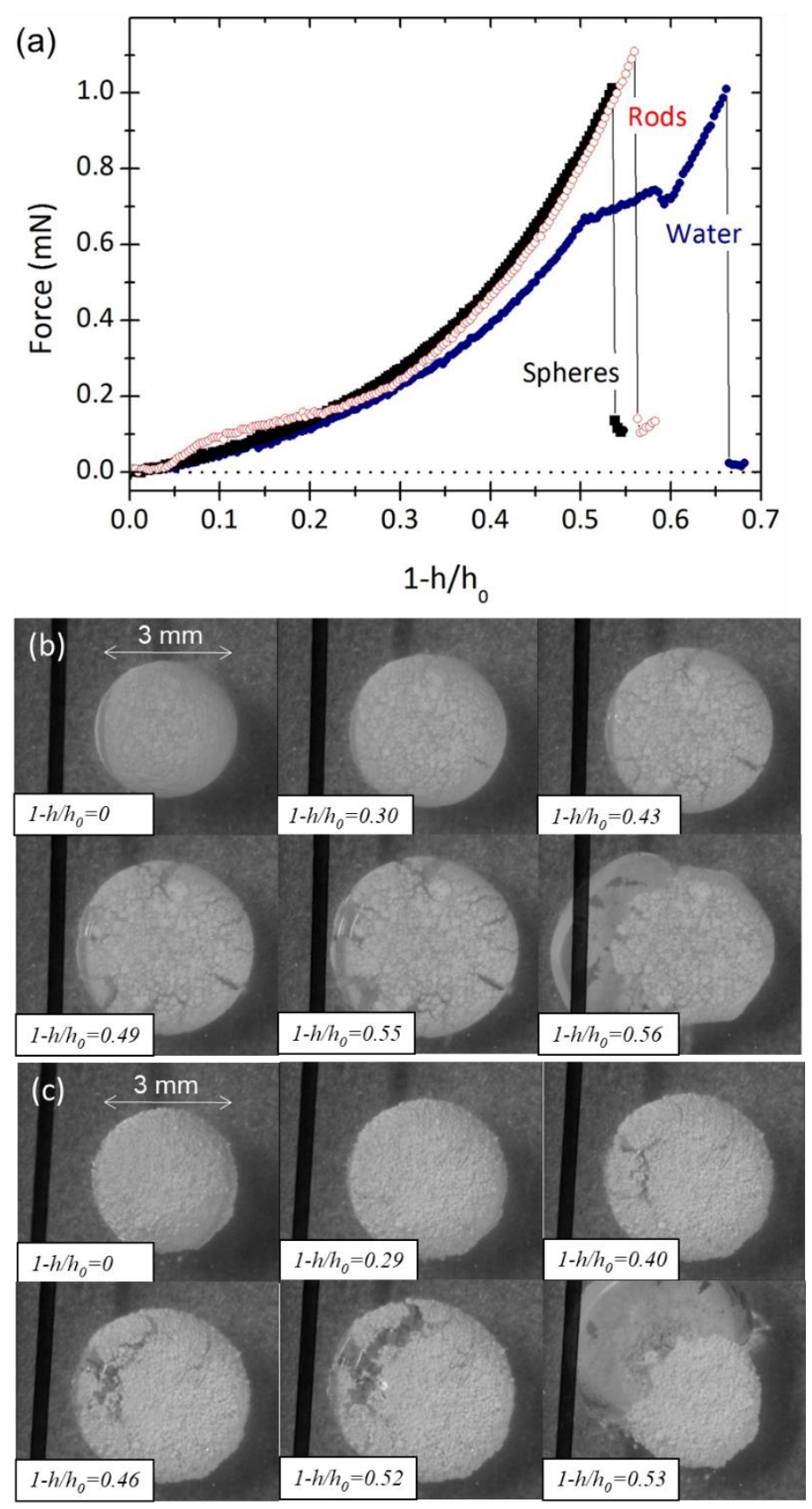

Figure 2. (a) The compression force on liquid marbles stabilized by spherical (L1) and rod-like particles (L2) and water droplet versus relative compression. (b) and (c) Top view images of the compressed liquid marbles (L2) and (L1), respectively, at different relative compression taken through the compressing glass plate. 


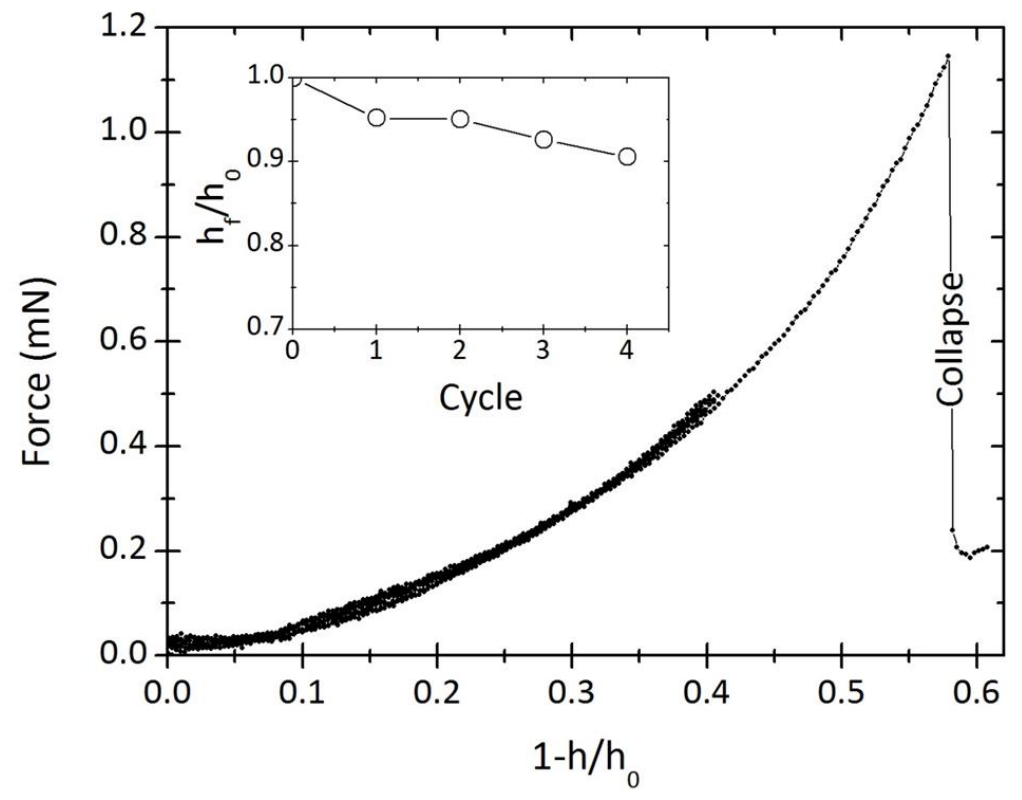

Figure 3. Compression-decompression force with the rate of $11 \mu \mathrm{m} / \mathrm{s}$ as a function of relative compression of liquid marble for different cycles. Inset: relative height of liquid marble (L1) in the uncompressed state after the $\mathrm{n}$-the cycle. 


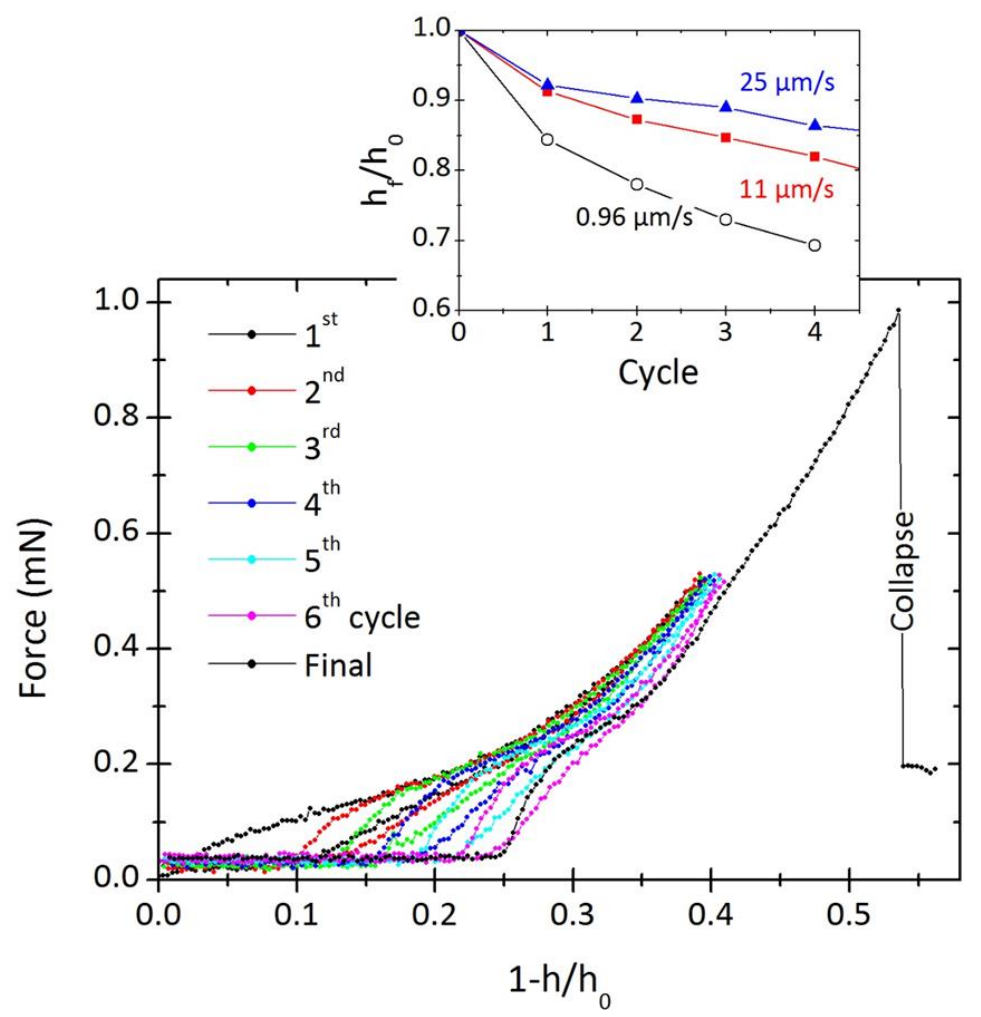

Figure 4. Compression-decompression force with the rate of $11 \mu \mathrm{m} / \mathrm{s}$ as a function of relative compression of liquid marble for different cycles. Inset: relative height of liquid marble (L2) after consecutive compression-decompression cycles and at different rates of compressiondecompression. 


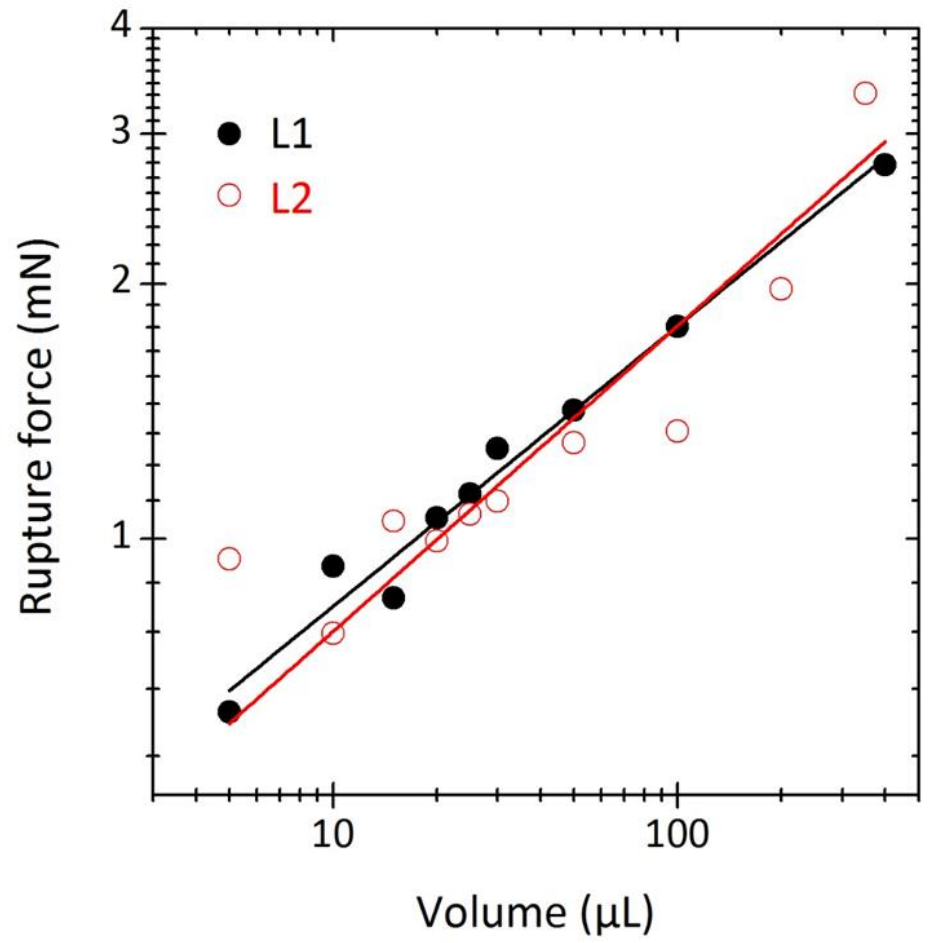

Figure 5. Plot of rupture force versus water volume of liquid marble for liquid marble L1 and liquid marble L2. 

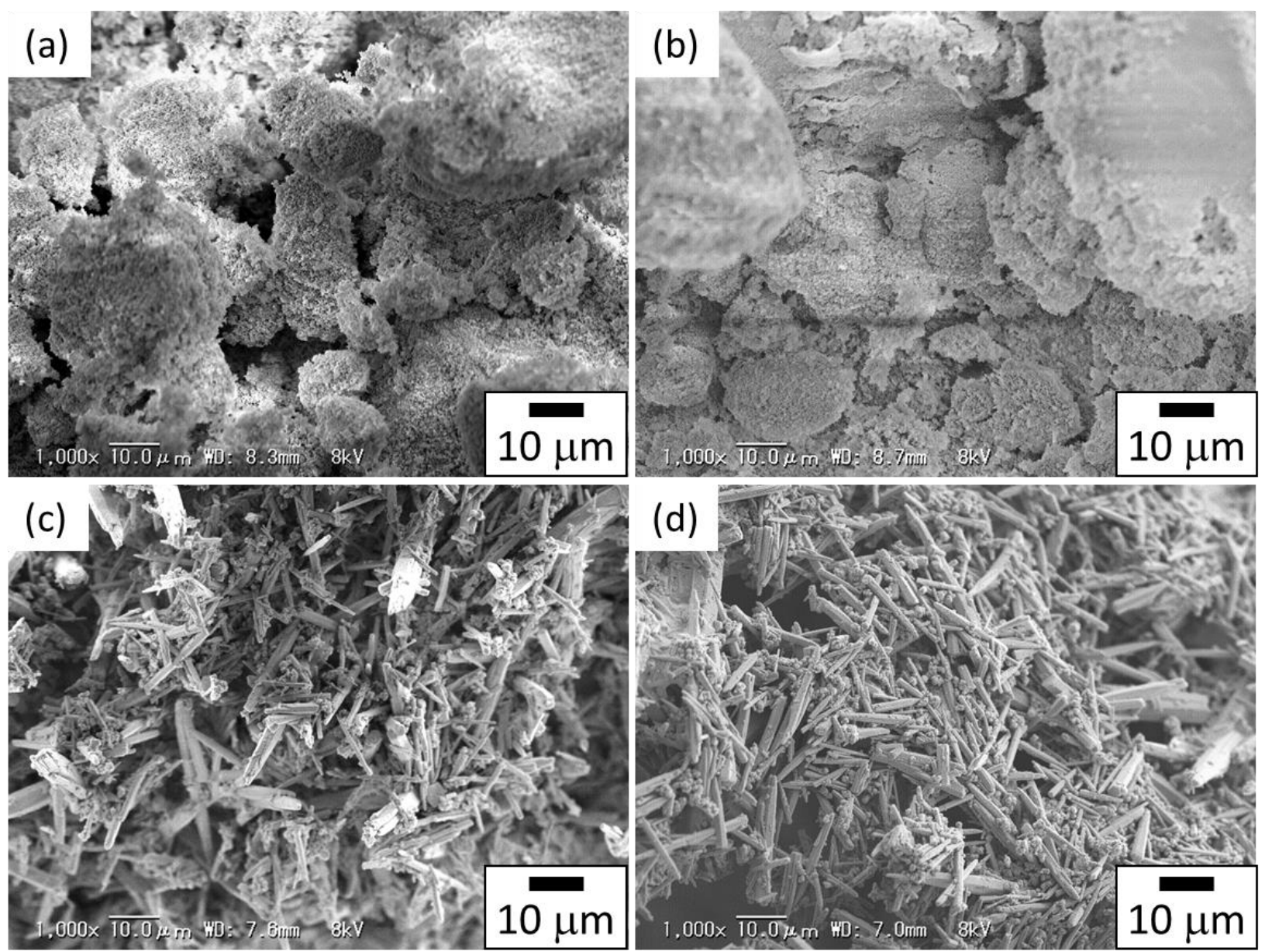

Figure 6. SEM images of surfaces of liquid marbles prepared using aqueous solution of Gellan gum as an inner liquid and $\mathrm{CaCO}_{3}$ nanoparticles as a stabilizer: (a,b) spherical $\mathrm{CaCO}_{3}$ and (c,d) rod-shaped $\mathrm{CaCO}_{3}$. The images were obtained $(\mathrm{a}, \mathrm{c})$ before and $(b, d)$ after compression. 


\section{ACKNOWLEDGMENTS}

S. Azizian acknowledges MPIP and Bu-Ali Sina University for financial support during the research visit. Shiraishi Kogyo Kaisha; Ltd. (Japan) and CPKelco (USA) were thanked for kind donations of $\mathrm{CaCO}_{3}$ nanoparticles and Gellan gum, respectively. This work was partially supported by JSPS-DAAD (Germany) Bilateral Joint Research Projects. Financial support from the ERC Grant No. 340391 SuPro (H. J. B.) is acknowledged,

\section{Appendix A. Supplementary materials}

Supplementary data associated with this article can be found in online version at http://dx.doi.org/. 


\section{REFERENCES}

1. P. Aussillous, D. Quéré, Properties of Liquid Marbles, Proc. R. Soc. A, 462, (2006), pp. 973999.

2. E. Bormashenko, Liquid Marbles: Properties and Applications, Curr. Opin. Colloid Interface Sci., 16, (2011), pp. 266-271.

3. E. Bormashenko, Liquid Marbles, Elastic Nonstick Droplets: From Minireactors to SelfPropulsion, Langmuir, 33, (2017), pp. 663-669.

4. G. McHale, M. I. Newton, Liquid marbles: Topical context within soft matter and recent progress, Soft Matter., 11, (2015), pp. 2530-2546.

5. C. H. Ooi, N.-T., Nguyen, Manipulation of liquid marbles, Microfluid Nanofluid, 19, (2015), pp. 483-495.

6. S. Fujii, S. Yusa, Y. Nakamura, Stimuli-Responsive Liquid Marbles: Controlling Structure, Shape, Stability, and Motion, Adv. Funct. Mater., 26, (2016), pp. 7206-7223.

7. Nuno M. Oliveira, Rui L. Reis, João F. Mano, The Potential of Liquid Marbles for Biomedical Applications: A Critical Review, Adv. Healthcare Mater. (2017), pp. 1700192(1)-1700192(15).

8. K. M. Lahanas, N. Vrabie, E. Santos, S. Miklean, Powder to Liquid Compositions, US $6290941 \mathrm{~B} 1,(2001)$.

9. C. Dampeirou, Hydrophobic Silica-based Water Powder, WO2005034917, (2005). 
10. M. I. Newton, D. L. Herbertson, S. J. Elliott, N. J. Shirtcliffe, G. McHale, Electrowetting of Liquid Marbles, J. Phys. D: Appl. Phys., 40, ( 2007), pp. 20-24.

11. M. Paven, H. Mayama, T. Sekido, H.-J. Butt, Y. Nakamura, S. Fujii, Light-Driven Delivery and Release of Materials Using Liquid Marbles, Adv. Funct. Mater., 26, (2016), pp. 3199-3206.

12. X. Lin, W. Ma, H. Wu, S. Cao, L. Huang, L. Chen, A. Takahara, Superhydrophobic Magnetic Poly(DOPAm-co-PFOEA)/Fe ${ }_{3} \mathrm{O}_{4} /$ cellulose Microspheres for Stable Liquid Marbles. Chem. Comm., 52, (2016), pp. 1895-1898.

13. Y. Xue, H. Wang, Y. Zhao, L. Dai, L. Feng, X. Wang, T. Lin, Magnetic Liquid Marbles: a "Precise" Miniature Reactor., Adv. Mater., 22, (2010), pp. 4814-4818.

14. T. Arbatan, A. Al-Abboodi, F. Sarvi, P. P. Y. Chan, W. Shen, Tumor Inside a Pearl Drop, Adv. Healthcare Mater., 1, (2012), pp. 467-469.

15. E. Sato, M. Yuri, S. Fujii, T. Nishiyama, Y. Nakamura, H. Horibe, Liquid Marbles as a Micro-reactor for Efficient Radical Alternating Copolymerization of Diene Monomer and Oxygen, Chem. Comm., 51, (2015), pp. 17241-17244.

16. T. Arbatan, L. Li, T. Tian, W. Shen, Liquid Marbles as Micro-bioreactors for Rapid Blood Typing, Adv. Health. Mater., 1, (2012), pp. 80-83.

17. T. Tian, T. Arbatan, X. Li, W. Shen, Liquid Marble for Gas Sensing, Chem. Comm., 46, (2010), pp. 4734-4736.

18. H. Zeng, Y. Zhao, Dynamic Behavior of a Liquid Marble Based Accelerometer, Appl. Phys. Lett., 96, (2010), pp. 114104(1)-114104(3). 
19. S. Fujii, S. Sawada, S. Nakayama, M. Kappl, K. Ueno, K. Shitajima, H.-J. Butt, Y. Nakamura, Pressure-sensitive Adhesive Powder, Mater. Horiz., 3, (2016), pp. 47-52.

20. X. Tang, S.-Y. Tang, V. Sivan, W. Zhang, A. Mitchell, K. Kalantar-zadeh, K. Khoshmanesh, Photochemically Induced Motion of Liquid Metal Marbles, Appl. Phys. Lett. 103, (2013), pp. 174104(1)-174104(4).

21. C. P. Whitby, X. Bian, R. Sedey, Spontaneous liquid marble formation on packed porous beds, Soft Matter., 8, (2012), pp. 11336-11342.

22.D. Dupin, S. P. Armes, S. Fujii, Stimulus-Responsive Liquid Marble, J. Am. Chem. Soc. 131 (2009) pp. 5386-5387.

23.S. Fujii, S. Kameyama, D. Dupin, S. P.Armes, M. Suzaki, Y. Nakamura, pH-Responsive Liquid Marbles Stabilized with Poly(2-vinylpyridine) Particles, Soft Matter 6 (2010) pp. 635-640.

24. H. Wu, H. Watanabe, W. Ma, A. Fujimoto, T. Higuchi, K. Uesugi, A. Takeuchi, Y. Suzuki, H. Jinnai, A. Takahara, Robust Liquid Marbles Stabilized with Surface-Modified Halloysite Nanotubes, Langmuir, 29, (2013), pp. 14971-14975.

25. G. Whyman, E. Bormashenko, Interpretation of Elasticity of Liquid Marbles, J. Colloid Interface Sci., 457, (2015), pp. 148-151.

26. Z. Liu, Z. Fu, B. P. Binks, H. C. Shum, Mechanical Compression to Characterize the Robustness of Liquid Marbles, Langmuir, 31, (2015), pp. 11236-11242.

27. J. Liu, P. Zuo, Wetting and Elasto-plasticity Based Sculpture of Liquid Marbles, Eur. Phys. J. E, 39(17), (2016), pp. 1-6. 
28. S. Asare-Asher, J. N. Connor, R. Sedev, Elasticity of Liquid Marbles, J. Colloid Interface Sci., 449, (2015), pp. 341-346.

29. S. Rigopoulos, A. Jones, Modeling of Semibatch Agglomerative Gas-Liquid Precipitation of $\mathrm{CaCO}_{3}$ in a Bubble Column Reactor, Ind. Eng. Chem. Res., 42, (2003), pp. 6567-6575.

30. S. Kakaraniya, A. Gupta, A. Mehra, Reactive Precipitation in Gas-slurry Systems: $\mathrm{The} \mathrm{CO}_{2-}$ $\mathrm{Ca}(\mathrm{OH})_{2}-\mathrm{CaCO}_{3}$ System, Ind. Eng. Chem. Res., 46, (2007), pp. 3170-3179.

31. E. Bormashenko, R. Pogreb, G. Whyman, A. Musin, Surface Tension of Liquid Marbles, Colloids Surf. A: Physicochem. Eng. Asp., 351, (2009), pp. 78-82.

32. X. Deng, L. Mammen, H.-J. Butt, D. Vollmer, Candle Soot as a Template for a Transparent Robust Superamphiphobic Coating, Science, 335(6064), (2012), pp. 67-70.

33. P. S. Bhosale, M. V. Panchagnula, H. A. Stretz, Mechanically Robust Nanoparticle Stabilized Transparent Liquid Marbles, Appl. Phys. Lett., 93, (2008), pp. 034109(1)-034109(3). 